\title{
The time course of adaptations in thermoneutral maximal oxygen consumption following heat acclimation
}

\author{
Mark Waldron $^{1,2}$ (D) O. Jeffries ${ }^{3} \cdot$ J. Tallent ${ }^{4} \cdot$ S. Patterson ${ }^{4} \cdot$ V. Nevola ${ }^{5}$
}

Received: 22 March 2019 / Accepted: 24 August 2019 / Published online: 12 September 2019

(c) The Author(s) 2019

\begin{abstract}
Purpose This study investigated the effects of a 10-day heat acclimation (HA) programme on the time course of changes in thermoneutral maximal oxygen uptake $\left(\dot{V} \mathrm{O}_{2 \max }\right)$ during and up to 10 days post-HA.

Methods Twenty-two male cyclists were assigned to a HA or control (Con) training group following baseline ramp tests of thermoneutral $\dot{V} \mathrm{O}_{2 \max }$. Ten days of fixed-intensity (50\% baseline $\left.\dot{V} \mathrm{O}_{2 \max }\right)$ indoor cycling was performed in either $38.0{ }^{\circ} \mathrm{C}$ (HA) or $\sim 20^{\circ} \mathrm{C}$ (Con). $\dot{V} \mathrm{O}_{2 \max }$ was re-tested on HA days 5, 10 and post-HA days $1,2,3,4,5$ and 10.

Results $\dot{V} \mathrm{O}_{2 \max }$ initially declined across time in both groups during training $(P<0.05)$, before increasing in the post-HA period in both groups $(P<0.05)$. However, $\dot{V} \mathrm{O}_{2 \max }$ was higher than control by post-HA day 4 in the HA group $(P=0.046)$. Conclusions The non-linear time course of $\dot{V} \mathrm{O}_{2 \max }$ adaptation suggests that post-testing should be performed 96-h posttraining to identify the maximal change for most individuals. In preparation for training or testing, athletes can augment their aerobic power in thermoneutral environments by performing 10 days HA, but the full effects will manifest at varying stages of the post-HA period.
\end{abstract}

Keywords Thermal $\cdot$ Training $\cdot$ Cycling $\cdot$ Endurance $\cdot$ Heat acclimation $\cdot$ Maximal aerobic power

$\begin{array}{ll}\text { Abbreviations } \\ \mathrm{CO}_{2} & \text { Carbon dioxide } \\ \mathrm{Con} & \text { Control } \\ \mathrm{Tc} & \text { Core body temperature } \\ \mathrm{HA} & \text { Heat acclimation } \\ \mathrm{HR} & \text { Heart rate } \\ \mathrm{Hct} & \text { Hematocrit } \\ \mathrm{Hb} & \text { Haemoglobin } \\ \dot{\mathrm{VO}} & \text { Maximal oxygen uptake } \\ \mathrm{PV} & \text { Plasma volume }\end{array}$

Communicated by Narihiko Kondo.

Mark Waldron

mark.waldron@swansea.ac.uk

College of Engineering, Swansea University, Swansea, UK

2 School of Science and Technology, University of New England, Armidale, NSW, Australia

3 School of Biomedical Sciences, Newcastle University, Newcastle Upon Tyne, UK

4 Sport, Health and Applied Sciences, St Mary's University, London, UK

5 Defence Science and Technology Laboratory (Dstl), Fareham, Hampshire, UK

$\begin{array}{ll}\text { RH } & \text { Relative humidity } \\ \text { RPE } & \text { Rating of perceived exertion } \\ \text { Ts } & \text { Thermal sensation }\end{array}$

\section{Introduction}

Heat acclimation (HA) describes a systematic process, whereby serial exposures to artificially heated environments, often in combination with exercise, can lead to rapid adaptations that enhance the capacity to thermoregulate in the heat (Gibson et al. 2015; Taylor and Cotter 2006). These adaptations improve heat tolerance (Sawka et al. 2011), characterised by increased sudomotor function (Fox et al. 1963), reduced heart rate (HR) (Senay et al. 1976), hypervolemia (Nielsen et al. 1993), increased cardiac output (Lorenzo et al. 2010) and a reduced core body temperature (Tc) for a given heat exposure or exercise intensity (Nielsen et al. 1993). This combination of physiological adaptations can enhance endurance performance in hot (Racinais et al. 2015) and thermoneutral environments (Lorenzo et al. 2010).

The maximal rate of oxygen uptake $\left(\dot{V} \mathrm{O}_{2 \max }\right)$ is an important determinant of endurance performance, explaining $\sim 20-60 \%$ of the variation in performances of different 
mode and distance (Coyle et al. 1988; Schabort et al. 2000; Jacobs et al. 2011). $\dot{V} \mathrm{O}_{2 \max }$ is chiefly limited by central cardiovascular factors, such as $\mathrm{O}_{2}$ transport (Bassett and Howley 2000). Heat acclimation can improve $\dot{V} \mathrm{O}_{2 \max }$, and is thought to occur owing to heat-induced cardiovascular changes (Périard et al. 2016). Indeed, there have been historical observations of a relationship between $\dot{V} \mathrm{O}_{2 \max }$ and heat tolerance (Shvartz et al. 1978; Pandolf 1979; Havenith and van Middendorp 1990). However, evidence of the efficacy of HA on $\dot{V} \mathrm{O}_{2 \max }$ is equivocal, with a number of studies demonstrating 4-13\% changes (Nadel et al. 1974; Shvartz et al. 1977; Sawka et al. 1985; Pivarnik et al. 1987; Lorenzo et al. 2010; James et al. 2017) and others reporting no change or a reduction following a range of HA protocols (Houmard et al. 1990; Gore et al. 1997; Chen et al. 2013; Karlsen et al. 2015; Keiser et al. 2015; Neal et al. 2016a, b; Rendell et al. 2017; Sotiridis et al. 2018).

There are various explanations for discrepancies in the aforementioned findings. First, not all studies have evaluated adaptations in $\dot{V} \mathrm{O}_{2 \max }$ in thermoneutral conditions, thus limiting the understanding of a transfer between heat-induced adaptation and aerobic capacity in temperate environments, which is a topic of ongoing debate (Nybo and Lundby 2016). This could be related to limited $\dot{V} \mathrm{O}_{2 \max }$ trainability (Bouchard et al. 2011) or inter-individual and inter-system differences in adaptation reported after 11 days of isothermal acclimation (Corbett et al. 2018). It is therefore possible that $\dot{V} \mathrm{O}_{2 \max }$ increases more substantially among HA-responsive individuals compared to their less responsive counterparts (Minson and Cotter 2016). Daanen et al. (2011) also demonstrated that the acute stress imposed by HA acted, initially, to suppress the adaptation in Tc, before adaptation beyond baseline in the days following acclimation. Thus, a period of recovery (from heat, exercise or both) might be necessary to fully realise adaptation in multi-system physiological measurements, such as $\dot{V} \mathrm{O}_{2 \max }$. This reasoning is consistent with the tenets of general adaptation syndrome (Selye 1950), which has been incorporated into exercise training guidelines (ACSM 2009). However, there has been no study to evaluate the detailed time course of change in thermoneutral $\dot{V} \mathrm{O}_{2 \max }$ after HA. Therefore, it is possible that previous studies have not: (1) monitored the inter-individual time course of responses of $\dot{V} \mathrm{O}_{2 \max }$ to HA across successive days and (2) provided adequate time for adaptation to the combined thermal and exercise training stimuli.

Based on this reasoning, we investigated the effects of a 10-day HA programme on the time course of changes in thermoneutral $\dot{V} \mathrm{O}_{2 \max }$, during and up to 10 days postHA against a control training group. We hypothesised that increases in $\dot{V} \mathrm{O}_{2 \max }$ would occur in the HA group in the days following the intervention but presumed variability between individuals in the time course of this response in the postHA 10-day period.

\section{Methods}

\section{Participants}

Twenty-two healthy, trained amateur male cyclists provided written informed consent to take part in this study. Twelve of the participants (age $23 \pm 3$ years, stature $1.77 \pm 0.61 \mathrm{~m}$, body mass $73.7 \pm 4.8 \mathrm{~kg}, \dot{V} \mathrm{O}_{2 \max } 60.8 \pm 6.1 \mathrm{ml} \mathrm{kg}^{-1} \mathrm{~min}^{-1}$ ) were randomly allocated to a $\mathrm{HA}$ group, while ten were allocated to a control group (age $25 \pm 3$ years, stature $1.78 \pm 0.46 \mathrm{~m}$, body mass $74.1 \pm 5.6 \mathrm{~kg}, \dot{V} \mathrm{O}_{2 \max } 59.8 \pm 6.7 \mathrm{ml} \mathrm{kg}^{-1} \mathrm{~min}^{-1}$ ). All of the participants were habitually training on a weekly basis $\left(13.8 \pm 3.3 \mathrm{~h} \cdot \mathrm{week}^{-1}\right)$ and competing in various amateur cycling events. All of the participants had taken part in outdoor hot weather training in the previous 12 months, while 16 had previously taken part in heat acclimation interventions for varying periods of time over the previous 3 years. However, all participants were deemed to be unacclimatised to heat stress within this study's HA protocol and they were habitually exposed to only the local environmental conditions or were at least 3 months without exposure. All participants completed a food diary for 2 days prior to each test, which was replicated with similar content and volume for the remainder of the study. The daily average high and low temperatures for the 6 weeks before and during all tests were 12.5 and $3.0^{\circ} \mathrm{C}$, respectively (accuweather.com). The participants were instructed not to use saunas or take hot baths during the study period. Participants were asked to refrain from alcohol and any supplementation during the study period and arrive at the laboratory having eaten a standardised meal and consumed $500 \mathrm{ml}$ of fluid in the previous $2 \mathrm{~h}$. The hydration and pre-meal were chosen by the participants, which we ensured were consistent between visits. Euhydration was verified via urine analysis using an osmometer $\left(<600 \mathrm{mOsmol} \mathrm{kg}{ }^{-1} \mathrm{H}_{2} \mathrm{O}\right.$, Osmocheck, Vitech Scientific Ltd, UK). Ethical approval was provided by the institutional ethics committee, which was conducted in accordance with the 1964 Helsinki Declaration.

\section{Design}

This study followed an independent groups design (HA vs. Con). After baseline tests of thermoneutral $\dot{V} \mathrm{O}_{2 \text { max }}$, the participants were randomly allocated to their groups using a Microsoft Excel random number generator. The HA group visited the laboratory for baseline measurements of $\dot{V} \mathrm{O}_{2 \max }$ and completed 10 days of 60 -min fixed-intensity $(50 \% \dot{V}$ $\left.\mathrm{O}_{2 \max }\right) \mathrm{HA}$ at $\left(38.0 \pm 2.4{ }^{\circ} \mathrm{C}, 30 \pm 13 \% \mathrm{RH}\right)$ in the following 10 -day period. The control group visited the laboratory on the same number of occasions but completed their exercise at the same intensity in controlled conditions $\left(20.0 \pm 1.1^{\circ} \mathrm{C}\right.$, $30 \pm 4 \% \mathrm{RH})$. On days 5,10 and post-days $1,2,3,4,5$ and 
10 , thermoneutral $\left(\sim 20^{\circ} \mathrm{C}\right) \dot{V} \mathrm{O}_{2 \max }$ tests (incremental ramp) were performed to assess the time course of changes during and after HA. Cycling training was continued by all of the participants during the study period, with the volume (mean of $60 \mathrm{~min} \cdot \mathrm{day}^{-1}$ ) subtracted from their normal regime. The participants' training during the study ranged across 3-4 separate sessions, comprising 2-3 long, lower-intensity rides $(>4 \mathrm{~h})$ and 1 higher-intensity interval session. None of the participants cross-trained, with all sessions performed in- or outdoors on a bicycle. In the post-HA period, no training was performed.

\section{Incremental ramp tests for $\dot{V} \mathrm{O}_{2 \max }$}

Participants were familiarised with the cycle ergometer (Monark Exercise AB, Ergomedic 874E, Varberg, Sweden) and saddle and handlebar position were recorded and repeated for all subsequent visits. Participants then completed a 5-min self-selected warm-up prior to completing an incremental ramp test. The test was conducted at self-selected cadences (range 70-95 rev $\mathrm{min}^{-1}$ ), starting at $\sim 120 \mathrm{~W}$ and increasing by $28-30 \mathrm{~W} \mathrm{~min}^{-1}$ until volitional exhaustion. The same increments were used across all trials, with a mean time to exhaustion of $8.5 \pm 1.8 \mathrm{~min}$. Pulmonary gas was measured continuously using a breath-by-breath gas analyser (Jaegar, Oxycon Pro, Viasys Healthcare, Hoechberg, Germany). The gas analyser and flow turbine were calibrated before each test using a known gas mixture $\left(15 \% \mathrm{O}_{2}\right.$ and 5\% $\mathrm{CO}_{2}$ ) and a 3-1 syringe, respectively (Hans Rudolph, Kansas City, KS). $\dot{V} \mathrm{O}_{2 \max }$ was determined as the mean value recorded over the final $30 \mathrm{~s}$ of the test. Criteria for achieving $\dot{V} \mathrm{O}_{2 \max }$ was: (1) reaching volitional exhaustion, (2) respiratory exchange ratio $>1.15$, (3) final HR within 10 beats $\mathrm{min}^{-1}$ of age-predicted maximum and (4) RPE $>19$. All criteria were met during the study. The same gas analyser was used throughout the study and calibrated identically. HR was recorded throughout the tests (Polar FT1, Polar Electro Oy, Kempele, Finland). End-power output was measured as the highest external power output reached during the final $1 \mathrm{~min}$ of the test. All tests were performed in the morning, prior to HA or thermoneutral training session (Con). In our laboratory, incremental tests of $\dot{V} \mathrm{O}_{2 \max }$ have a CV\% of $3.0 \%$. The first three $\dot{V} \mathrm{O}_{2 \text { max }}$ tests were performed prior to the HA session for that day, in a thermoneutral environment, at the same time of day.

\section{Heat acclimation protocol}

The power output corresponding to $50 \%$ of the participants' baseline $\dot{V} \mathrm{O}_{2 \max }$ was set as the external work intensity for the intervention and was monitored using power output on the cycle ergometer. Cadence was self-selected and adjusted if necessary by the investigators using weights to maintain the target intensity. This intensity was maintained for all subsequent trials but was reduced by $10 \%$ if it could not be sustained by the participant. Participants cycled for $60 \mathrm{~min}$ per session. This type of HA protocol was selected based on previous studies (Houmard et al. 1990; Senay et al. 1976; Lorenzo et al. 2010; Pandolf et al. 1977). The participants' nude body mass was recorded pre- and post-session on days 1,5 and 10 of the HA programme to estimate WBSR by subtracting post-exercise body mass from pre-exercise values (MPMS-230, Marsden Weighing Group, Oxfordshire, UK). A rectal thermometer (Edale Instruments Ltd, Cambridge, UK) was self-inserted $10 \mathrm{~cm}$ past the anal sphincter, as an indication of Tc, and recorded every 2 min via a scanning thermometer once inside the heat-controlled chamber (Edale Instruments Ltd, Cambridge, UK). The mean Tc recorded was used for statistical analysis. The participants then entered the heat chamber wearing cycling shorts, socks and training shoes, where they sat upright on the same cycle ergometer used during the ramp test. HR was also recorded, alongside thermal sensation (Ts) at 5-min intervals throughout the exercising protocol. Ts was recorded on an ASHRAE 7-point analogue scale, where $-3=$ "very cold", $0=$ "neutral", and $3=$ "very hot" (Zhang et al. 2004). In the control condition, the heat chamber was controlled at $20.0 \pm 1.1^{\circ} \mathrm{C}$, $30 \pm 4 \%$ RH. No fans were used during the exercise trials and no fluid intake was permitted until after the session when post-measures were taken.

\section{Plasma volume}

On arrival at the laboratory in the morning of HA day 1, HA day 5 and HA day 10 and post-HA day 10, the participants from both the HA and control groups rested in an upright seated position in an air-conditioned room $\left(20{ }^{\circ} \mathrm{C}\right.$ and $50 \%$ relative humidity) for $15 \mathrm{~min}$. Changes in the concentration of haematocrit (Hct) and haemoglobin $(\mathrm{Hb})$ were subsequently recorded to determine the relative change in plasma volume (Dill and Costill 1974). Capillary blood was drawn from the index finger into two $75 \mathrm{~mm}$ hematocrit capillary tubes for duplicate measurements. The whole blood was centrifuged (Hawksley Haematospin 1400 Centrifuge, Hawksley and Sons Ltd., Sussex, UK) for $5 \mathrm{~min}$ at 13,000g. Postcentrifugation, capillary tubes were analysed for[Hct using a micro-capillary reader (Hawksley and Sons Ltd., Sussex, UK), with the mean of the two measurements reported. All measurements agreed by less than $2 \%$. Capillary blood was taken from the same site for measurement of $\mathrm{Hb}$ using a Hemocue Hb $201+$ (Hemocue Ltd, Viking Court, Derbyshire, UK). Plasma volume changes $(\triangle \% \mathrm{PV})$ were reported between HA days 1 and 5, HA days 5 and 10 and HA day 10 and post-HA day 10 . 


\section{Statistical analysis}

Changes in $\dot{V} \mathrm{O}_{2 \max }$, end power $(2 \times 9[$ time] $), \Delta \%$ body mass and $\Delta \% \mathrm{PV}(2 \times 3$ [time] $), \mathrm{Tc}$, end $\mathrm{HR}$ and Ts recorded during the HA programme $(2 \times 10$ [time] $)$ were analysed using twoway (group $\times$ time) within- and between-analyses of variance. All of the participant characteristics were compared using independent $t$ tests, to check for baseline differences. Where relevant, assumptions of sphericity were assessed using Mauchly's test, with any violations adjusted using the Greenhouse-Geisser correction. When significant $F$ values were observed, post hoc tests were used to determine differences. Statistical significance was accepted at $P<0.05$ for all tests and all analyses were performed on IBM SPSS Statistics (Version 21, IBM Corp., Armonk, NY, USA).

\section{Results}

\section{Participant characteristics}

There were no differences $(P>0.05)$ in the characteristics of the HA and control groups (Table 1). Both groups of participants possessed high $\dot{V} \mathrm{O}_{2 \max }$ values relative to their age (Rapp et al. 2018), and followed cycling-specific programmes both habitually ( 13 to $14 \mathrm{~h} \mathrm{week}^{-1}$ ) and during the study ( 10 to $11 \mathrm{~h})$.

\section{$\dot{V} \mathrm{O}_{2 \max }$ and end-power output}

Changes in $\dot{V} \mathrm{O}_{2 \max }$ across the HA or control programmes are presented in Fig. 1. There was an effect of time on $\dot{V}$ $\mathrm{O}_{2 \max }\left[F_{(8,160)}=11.49, P<0.001\right]$, and group $\times$ time interactions $\left[F_{(8,160)}=5.20, P<0.001\right]$, with post hoc tests revealing reductions in $\dot{V} \mathrm{O}_{2 \max }$ between baseline and HA days 5 and 10 for the HA group $(P<0.001$ and $P=0.003$, respectively) and control $(P=0.029$ and $P=0.032$, respectively). However, only the HA group demonstrated reduction in $\dot{V} \mathrm{O}_{2 \max }$

Table 1 Participant characteristics for the heat acclimation (HA; $n=12)$ and control groups $(n=10)$

\begin{tabular}{lcc}
\hline & HA (mean \pm SD) & Control (mean \pm SD) \\
\hline Age (years) & $23 \pm 3$ & $25 \pm 2$ \\
Nude body mass $(\mathrm{kg})$ & $73.7 \pm 4.8$ & $74.1 \pm 5.6$ \\
$\dot{V} \mathrm{O}_{2 \mathrm{max}}\left(\mathrm{ml} \mathrm{kg}^{-1} \mathrm{~min}^{-1}\right)$ & $60.8 \pm 6.1$ & $59.8 \pm 6.7$ \\
Annual weekly training (h) & $14.0 \pm 2.8$ & $13.6 \pm 4.1$ \\
Previous acclimation & $9.7 \pm 9.1$ & $4.8 \pm 2.7$ \\
$\quad($ days) & & \\
Training during study (h) & $11.3 \pm 3.3$ & $10.3 \pm 2.7$ \\
Training age (years) & $4.1 \pm 1.8$ & $4.0 \pm 1.6$ \\
\hline
\end{tabular}

${ }^{\mathrm{a}}$ In the previous 3 years
A
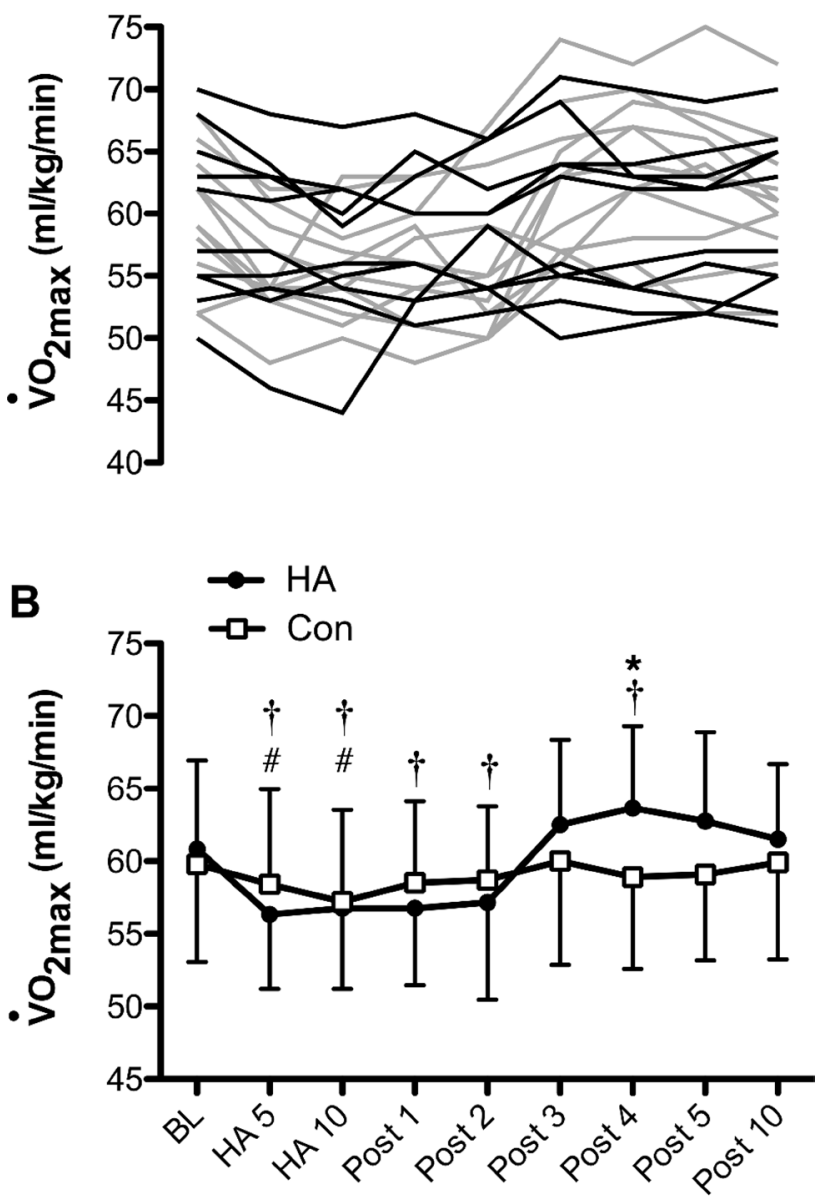

Testing stage

Fig. 1 a Individual maximal oxygen consumption $\left(\dot{V} \mathrm{O}_{2 \max }\right)$ responses in the HA group (grey) and control (black). b Changes in $\dot{V} \mathrm{O}_{2 \max }$ across the heat acclimation (HA, $n=12$ ) and control (Con, $n=10)$ interventions. *Difference $(P<0.05)$ between groups at that stage; ${ }^{\dagger}$ Sig. different $(P<0.05)$ to baseline (BL) for HA group; ${ }^{\#}$ sig. different $(P<0.05)$ to $\mathrm{BL}$ for Con group. Time $=P<0.001$; group $\times$ time $=P<0.001$

compared to baseline on post-HA day $1(P=0.004)$, post-HA day $2(P=0.008)$, with subsequent increase post-HA day 4 relative to baseline $(P=0.038)$. Across the post-HA period, there were no changes $(P>0.05)$ in $\dot{V} \mathrm{O}_{2 \max }$ relative to baseline for the control group. Furthermore, post hoc tests identified differences in $\dot{V} \mathrm{O}_{2 \max }$ between the HA group and control group only at post-HA day $4(P=0.046)$. As presented in Fig. 2, end-power output followed a similar pattern to $\dot{V} \mathrm{O}_{2 \max }$ measurements, with time effects $\left[F_{(8,160)}=13.24, P<0.001\right]$, characterised by reductions from BL on HA days 5,10 and post-HA days $1(P<0.05)$. End-power output increased from HA day 10 to post-HA days $3(P=0.004)$ and $4(P=0.004)$, with no further changes. There were interactions between 


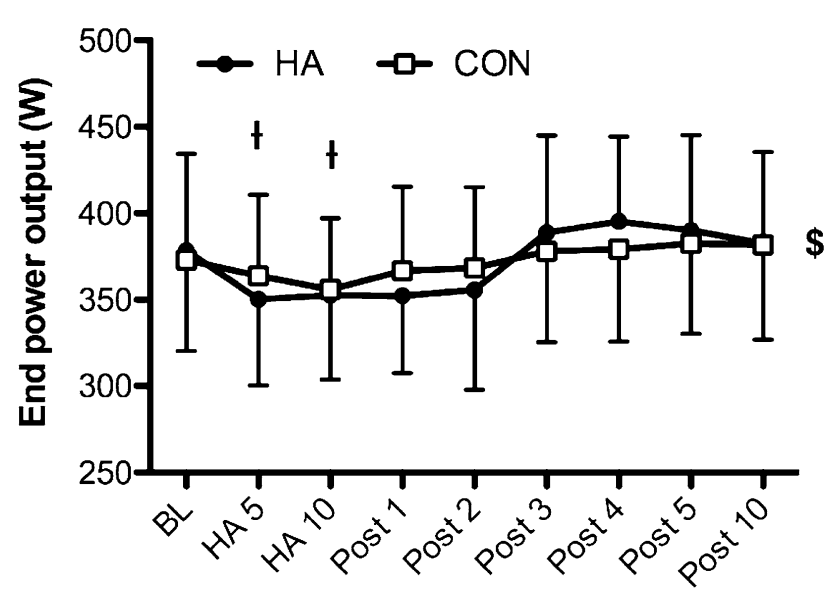

Testing stage

Fig. 2 Changes in end-power output across the heat acclimation (HA, $n=12)$ and control (Con, $n=10)$ interventions. ${ }^{\$}$ Main effect of time $(P<0.05)$. Time $=P<0.001 ;$ group $\times$ time $=P=0.024$. ${ }^{~}$ Pairwise time effect from baseline $(P<0.05)$

time and group $\left[F_{(8,160)}=2.33, P=0.024\right]$, but no pairwise effects were identified $(P>0.05)$.

\section{Core temperature, heart rate and thermal sensation}

There were time effects for mean $\operatorname{Tc}\left[F_{(9,180)}=26.93\right.$, $P<0.001]$, with step-wise reductions from day 1 across the three subsequent HA days $(P<0.05)$. The main contributions to the time effects were from the HA group, demonstrated via interactions $\left[F_{(9,180)}=11.71, P<0.001\right]$ and pairwise differences across all days $(P<0.05)$ (Fig. 3$)$. Mean heart rate $\left[F_{(9,180)}=38.90, P<0.001\right]$ and thermal sensation $\left[F_{(9,180)}=5.56, P<0.001\right]$ followed a similar pattern across time. Time and group interacted $\left[F_{(9,180)}=17.07, P<0.001\right]$ for heart rate responses, with pairwise differences across all HA days (Fig. 4). Similarly, group $\times$ time interactions $\left[F_{(9,180)}=2.69, P=0.045\right]$ were found for Ts and pairwise differences across all HA days $(P<0.05)$ (Fig. 5).

\section{Plasma volume and body mass changes}

The $\triangle \% \mathrm{PV}$ between $\mathrm{HA}$ days 1 and 5 was larger $(P<0.001)$ in the HA group compared to control $(8.8 \pm 5.0 \%$ vs. $0.7 \pm 0.8 \%$, respectively $)$. There were no further between-group differences $(P>0.05)$ in $\Delta \% \mathrm{PV}$, with the HA group maintaining their initial PV expansion between HA days 5 and 10 (HA $1.1 \pm 2.6 \%$ vs. Con $-1.2 \pm 1.3 \%$ ) and HA day 10 and post-HA day 10 (HA $0.6 \pm 2.1 \%$ vs. Con $-1.11 \pm 1.5 \%)$. There was an effect of group for $\Delta \%$ body mass changes $\left[F_{(1,20)}=30.05\right.$,

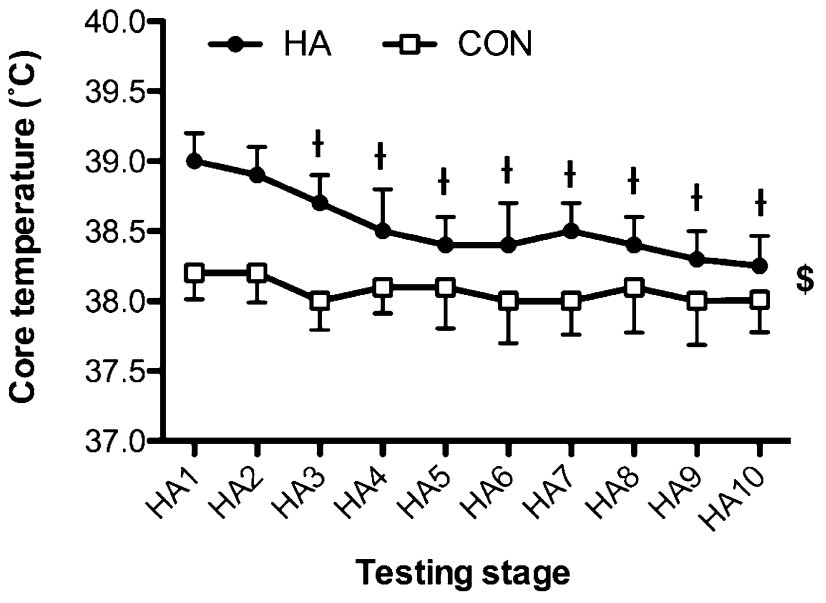

Fig. 3 Changes in core temperature across the heat acclimation (HA, $n=12$ ) and control (Con, $n=10)$ days. $\$=$ main effect of time $(P<0.05)$. Differences $(P<0.05)$ were found between groups at each stage but not noted for clarity. Time $=P<0.001$; group $\times$ time $=P<0.001$. 'Pairwise time effect from baseline $(P<0.05)$

$P<0.001]$, denoting greater WBSR in the HA group, but no interactions with time $\left[F_{(2,40)}=0.25, P=0.068\right]$.

\section{Discussion}

We are the first to evaluate the time course of changes in thermoneutral $\dot{V} \mathrm{O}_{2 \max }$ adaptation during and after a HA programme. Changes in $\dot{V} \mathrm{O}_{2 \max }$ were elicited in a non-linear manner during the 20-day (combined HA and post-HA)

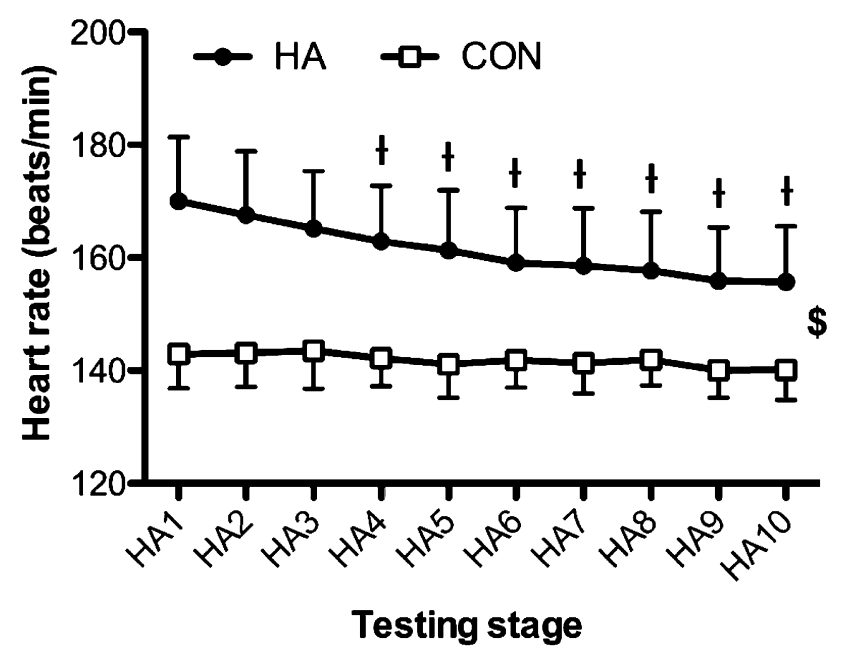

Fig. 4 Changes in mean heart rate responses across the heat acclimation (HA, $n=12)$ and control (Con, $n=10)$ days. $\$=$ main effect of time $(P<0.05)$. Differences $(P<0.05)$ were found between groups at each stage but not noted for clarity. Time $=P<0.001$; group $\times$ time $=P<0.001$. ${ }^{\mathrm{t}}$ Pairwise time effect from baseline $(P<0.05)$ 


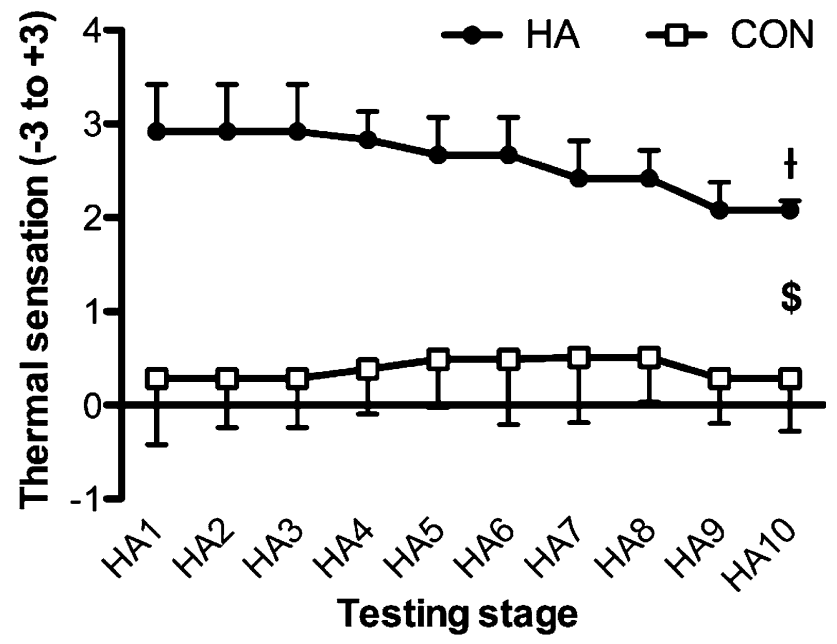

Fig. 5 Changes in thermal sensation responses across the heat acclimation (HA, $n=12)$ and control (Con, $n=10)$ days. ${ }^{\$}$ Main effect of time $(P<0.05)$. Differences $(P<0.05)$ were found between groups at each stage but not noted for clarity. Time $=P<0.001$; group $\times$ time $=P=0.045$. 'Pairwise time effect from baseline $(P<0.05)$

period, with acute reductions apparent at HA days 5 and 10 among both groups. Delayed increase in thermoneutral $\dot{V} \mathrm{O}_{2 \max }$ occurred in the post-HA (recovery) period. Specifically, by post-HA day 4 , there were pairwise differences between the HA and control groups, where mean changes in $\dot{V} \mathrm{O}_{2 \max }$ peaked (4.9\% increase from baseline) for the HA group. The control group's $\dot{V} \mathrm{O}_{2 \max }$ fluctuated between -4.5 and $0.3 \%$ approximating the $3.0 \%$ error of the test. There were notable inter-individual differences in the time course of adaptation among the HA group, yet only 1 of the 12 participants did not respond to the HA programme, demonstrating their highest value prior to the post-HA period. These adaptations were supported by larger plasma volume expansion in the HA group, which was maintained across the study period.

There are a number of studies demonstrating HAinduced increase in thermoneutral $\dot{V} \mathrm{O}_{2 \max }$, ranging between 4 and 13\% (Nadel et al. 1974; Shvartz et al. 1977; Sawka et al. 1985; Pivarnik et al. 1987; Lorenzo et al. 2010; James et al. 2017). Similarly, we report a 4.9\% increase on post-HA day 4 compared to baseline, which is substantial, given the limited potential for adaptation in $\dot{V}$ $\mathrm{O}_{2 \max }$ (Bouchard et al. 2011) and the trained status of the participants. Furthermore, this value was increased to a mean of $7.1 \%$ if the largest individual changes in $\dot{V} \mathrm{O}_{2 \max }$ observed across the study are accounted for. These data suggest that the changes reported previously are likely to have underestimated the full adaptation potential, owing to inter-individual variability in this measure. Our findings highlight that the timing of the post-acclimation testing is crucial to the outcome, despite most studies not precisely reporting this. Based on the current data, we recommend that post-acclimation $\dot{V} \mathrm{O}_{2 \max }$ testing is not performed within the post-HA $48 \mathrm{~h}$ and that a $96-\mathrm{h}$ posttest is likely to produce the highest value for most individuals. Of note, the one participant's $\dot{V} \mathrm{O}_{2 \max }$ that did not peak in the post-HA period was highest at baseline and remained lower for the entire 20-day period. This denotes an important individual feature of training adaptations and questions rigid training models. As reported elsewhere, it is also possible that some individuals experience a delayed response (absence of decay) to heat acclimation (Daanen et al. 2011) of up to 26 days (Weller et al. 2007), which would not have been captured in our 10-day post-HA window and is one explanation for the participant's response in the current study. This descriptive analysis provides insight into the dynamics of heat-induced adaptation in $\dot{V} \mathrm{O}_{2 \max }$ between individuals and could lead to imprecise reporting in studies of this type, depending on the selected post-testing period.

As noted previously (Armstrong and Maresh 1991; Nielsen et al. 1993), there were some characteristic signs of early adaptation demonstrated across the sub-maximal HA sessions, with Tc, HR and Ts declining across the first 3 days in the HA group. These changes indicate an improved tolerance to the exercise and thermal stimuli. However, the time course of $\dot{V} \mathrm{O}_{2 \max }$ adaptation to HA was not as immediate and, therefore, did not coincide with early adaptations in Tc, HR and Ts. This is important to recognise because HA-induced changes in $\dot{V} \mathrm{O}_{2 \max }$ are typically ascribed to central cardiovascular adaptations that are responsible for $\mathrm{O}_{2}$ transport. For example, $\dot{V} \mathrm{O}_{2 \max }$ is chiefly determined by the delivery of oxygenated blood to the working musculature and relies on factors, such as stroke volume and muscle capillarisation, to support this (Bassett and Howley 2000). The lower HR responses to the HA programme reported here and elsewhere (Nielsen et al. 1993; Lorenzo et al. 2010), and increased plasma volume, are consistent with an improved cardiac efficiency, which is commonly observed following HA (Périard et al. 2016). These changes also infer an increase in stroke volume and cardiac output (Senay et al. 1976; Nielsen et al. 1997). Thus, the temporal differences in $\dot{V} \mathrm{O}_{2 \max }$ adaptation, highlighted by acute decline in $\dot{V} \mathrm{O}_{2 \max }$, must be attributed to other factors in the $\mathrm{O}_{2}$ transport pathway, perhaps indicating an acute impairment and perfusion capacity of muscle microvasculature function during the HA programme. There have been reports of increased vascular conductance after 10-14 days of HA in the peripheral cutaneous microvasculature (Lorenzo and Minson 2010) and others have reported changes in $\mathrm{O}_{2}$ pulse at high exercise intensities, which were ascribed to increase in arterial-venous $\mathrm{O}_{2}$ differences (Chen et al. 2013). Together, these findings indicate that HA induces an increase in peripheral blood flow that could lead to enhanced 
perfusion of blood in the working muscles. However, there has been no study of the time course of this adaptation to monitor early-phase changes in the microvasculature of the skeletal muscle. Further work is required to elucidate this.

$\dot{V} \mathrm{O}_{2 \max }$ relies on multiple physiological factors; therefore, a less predictable time course of adaptation might have been anticipated. General adaptation syndrome describes a process of systematic overload to impose controlled 'stress', leading to changes in that system, such that subsequent exposures to the same stress are more tolerable (Selye 1950). Indeed, the discordant temporal adaptations between different physiological processes observed here are supported by Selye's (1938) concept of 'adaptation energy', whereby simultaneous adaptations to given stimuli are constrained by finite energy resources, which was originally demonstrated by exposing rats to cold temperatures and exercise. Similar adaptation kinetics have been demonstrated in the exercise training literature, where 1-3 weeks of overreaching in cyclists acutely reduced $\dot{V} \mathrm{O}_{2 \max }$ prior to rebound improvements in the recovery period (Jeukendrup et al. 1992; Aubry et al. 2014). These changes have not been fully explained but attributed to a general training fatigue or psychological factors that ensue during heavy training periods. This theory has also been previously applied to the process of HA, where Daanen et al. (2011) reported lower resting and exercising $\mathrm{Tc}-\mathrm{a}$ principal feature of adaptation to heat exposure-to manifest most prominently during the postHA period. This had been reported in other investigations (Pandolf et al. 1977) and was explained by the severity of the imposed thermal stimulus. It is plausible that the same reasoning applies to the current data, since the participants trained for the duration of the study, in addition to the thermal load and serial $\dot{V} \mathrm{O}_{2 \max }$ testing. However, the absence of significant $\dot{V} \mathrm{O}_{2 \max }$ changes over time in the control group or differences in training characteristics compared to the HA group suggests that the thermal stimulus was the responsible factor. Alternatively, it is possible that kinetics of $\dot{V} \mathrm{O}_{2 \max }$ adaptation are different to other measures but has not yet been considered with the necessary scrutiny.

The suggestion that $\dot{V} \mathrm{O}_{2 \max }$ adaptation occurs in the post-HA period is at odds with the characteristic rapid decay reported in other physiological measures, such as Tc and HR (Garrett et al. 2009, 2011; Daanen et al. 2018). Indeed, it is the concern over rapid post-HA decay that has most likely prompted testing in the following 72-h period (i.e., Sotiridis et al. 2018). Of note, some studies have reported descriptive increase in $\dot{V} \mathrm{O}_{2 \max }$ of up to $3.5 \%$ and significant increase in end test power of $8.5 \%$ (Sotiridis et al. 2018), yet re-tested across 3 consecutive days, immediately following HA. Based on the current data, $\dot{V} \mathrm{O}_{2 \max }$ does not appear to enter a period of sustained 'decay' for the first 4 days, and perhaps up to 10 days, after HA. Future studies should focus on manipulating the intensity of the stimulus and extend the period of post-HA monitoring to establish the full time course of decay in $\dot{V}$ $\mathrm{O}_{2 \max }$. In this regard, the chosen HA protocol followed a so-called 'fixed-intensity' model, which is not thought to provide an optimal stimulus for adaptation to the heat, as Tc is uncontrolled by the investigator (Daanen et al. 2018). Indeed, others have suggested that acclimation $>38.5{ }^{\circ} \mathrm{C}$ Tc is necessary for adaptation (Fox et al. 1963). However, recent studies have questioned this recommendation, reporting no relationship between thermal load (time spent $>38.5^{\circ} \mathrm{C}$ ) and changes in Tc or HR (Corbett et al. 2018). Others have used fixed-intensity HA programmes to induce changes in $\dot{V} \mathrm{O}_{2 \max }$ (Lorenzo et al. 2010) and more recent studies have demonstrated similar heat adaptations between isothermal or fixed-intensity approaches (Gibson et al. 2015). There are some potential advantages to adopting a fixed-intensity protocol. For example, the absolute power output and relative intensity sustained in a fixedintensity protocol is higher than reported in isothermal models (Gibson et al. 2015), which might be of greater importance when a gross outcome $\left(\dot{V} \mathrm{O}_{2 \max }\right)$ is targeted, requiring a mixture of thermal and exercise stimuli. The Tc and HR responses were also significantly higher than the control group across all sessions, with mean Tc values beginning at a mean of $39{ }^{\circ} \mathrm{C}$ and finishing marginally above $38.2{ }^{\circ} \mathrm{C}$ on day 10 . Furthermore, this model can be practically simpler to run with groups of athletes, which is often necessary for applied practitioners. Therefore, the approach we have adopted appears to have provided sufficient stimulus for adaptation in $\dot{V} \mathrm{O}_{2 \max }$ and the delayed increase could, theoretically, suit the needs of individuals who cannot acclimate for the entire pre-competition training camp, owing to travel arrangements or the details of their pre-event taper.

There are some limitations to the current study, such as the types of athletes used, who were all cyclists and well trained. While we do not anticipate that delayed $\dot{V} \mathrm{O}_{2 \max }$ adaptation will be specific to the mode of exercise, it is possible that less trained participants would demonstrate a different time course of adaptation to training stimuli and that the lower aerobic capacity might prevent them from fully engaging in the intensive acclimation process. Furthermore, all of the cyclists underwent an acclimation intervention, rather than acclimatisation, where there is typically less control over the ambient conditions (i.e., heat, humidity and wind speed). Thus, we cannot be certain that the same effects would be observed in a natural environment. 


\section{Conclusion}

The time course of $\dot{V} \mathrm{O}_{2 \max }$ adaptation is non-uniform, mimicking a typical supercompensation response. These findings have implications for researchers and athletes wishing to evaluate adaptations in $\dot{V} \mathrm{O}_{2 \max }$ after performing HA programmes. We advise that post-testing is not performed in the following $48 \mathrm{~h}$ and that $96 \mathrm{~h}$ provides the peak adaptation for most individuals. Whilst the current findings are of fundamental scientific interest, there are also many practical advantages of using heat acclimation to enhance thermoneutral aerobic capacity. This includes the ability to induce a variety of physiological adaptations (reduced Tc and HR, increased sweat response), alongside changes in $\dot{V} \mathrm{O}_{2 \max }$, in response to a training programme.

Author contributions MW conceived and designed research. MW, OJ, SP, JT and RN conducted experiments, analyzed data and wrote the manuscript. All authors read and approved the manuscript.

\section{Compliance with ethical standards}

Conflict of interest The authors declare that they have no conflict of interest.

Ethical approval All procedures were performed in accordance with the ethical standards of the institutional and/or national research committee and with the 1964 Helsinki Declaration and its later amendments or comparable ethical standards.

Informed consent Informed consent was obtained from all individual participants included in the study.

Open Access This article is distributed under the terms of the Creative Commons Attribution 4.0 International License (http://creativeco mmons.org/licenses/by/4.0/), which permits unrestricted use, distribution, and reproduction in any medium, provided you give appropriate credit to the original author(s) and the source, provide a link to the Creative Commons license, and indicate if changes were made.

\section{References}

American College of Sports Medicine (2009) American College of Sports Medicine Position Stand. Progression models in resistance training for healthy adults. Med Sci Sports Exerc 41:687-708

Armstrong LE, Maresh CM (1991) The induction and decay of heat acclimatisation in trained athletes. Sports Med 12:302-312

Aubry A, Hausswirth C, Louis J, Coutts AJ, Le Meur Y (2014) Functional overreaching: the key to peak performance during the taper? Med Sci Sports Exerc 46:1769-1777

Bassett DR, Howley ET (2000) Limiting factors for maximum oxygen uptake and determinants of endurance performance. Med Sci Sports Exerc 32:70-84

Bouchard C, Sarzynski MA, Rice TK, Kraus WE, Church TS, Sung YJ, Rao DC, Rankinen T (2011) Genomic predictors of the maximal
$\mathrm{O}_{2}$ uptake response to standardized exercise training programs. J Appl Physiol 110:1160-1170

Chen TI, Tsai PH, Lin JH, Lee NY, Liang MT (2013) Effect of shortterm heat acclimation on endurance time and skin blood flow in trained athletes. Open Access J Sports Med 18:161-170

Corbett J, Rendell RA, Massey HC, Costello JT, Tipton MJ (2018) Inter-individual variation in the adaptive response to heat acclimation. J Therm Biol 74:29-36

Coyle EF, Coggan AR, Hopper MK, Walters TJ (1988) Determinants of endurance in well-trained cyclists. J Appl Physiol 64:2622-2630

Daanen HAM, Jonkman AG, Layden JD, Linnane DM, Weller AS (2011) Optimising the acquisition and retention of heat acclimation. Int J Sports Med 32:822-828

Daanen HAM, Racinais S, Périard J (2018) Heat acclimation decay and re-induction: a systematic review and meta-analysis. Sports Med 48:409-430

Dill DB, Costill DL (1974) Calculation of percentage changes in volumes of blood, plasma, and red cells in dehydration. J Appl Physiol 37:247-248

Fox RH, Goldsmith R, Kidd DJ, Lewis HE (1963) Acclimatization to heat in man by controlled elevation of body temperature. J Physiol 166:530-547

Garrett AT, Goosens NG, Rehrer NJ, Patterson MJ, Cotter JD (2009) Induction and decay of short-term heat acclimation. Eur J Appl Physiol 107:659-670

Garrett AT, Rehrer NJ, Patterson MJ (2011) Induction and decay of short-term heat acclimation in moderately and highly trained athletes. Sports Med 41:757-771

Gibson OR, Mee JA, Tuttle JA, Taylor L, Watt PW, Maxwell NS (2015) Isothermic and fixed intensity heat acclimation methods induce similar heat adaptation following short and long-term timescales. J Therm Biol 49:55-65

Gore CJ, Hahn AG, Burge CM, Telford RD (1997) $\mathrm{VO}_{2 \max }$ and haemoglobin mass of trained athletes during high intensity training. Int J Sports Med 18:477-482

Havenith G, van Middendorp H (1990) The relative influence of physical fitness, acclimatization state, anthropometric measures and gender on individual reactions to heat stress. Eur J Appl Physiol Occup Physiol 61:419-427

Houmard JA, Costill DL, Davis JA, Mitchell JB, Pascoe DD, Robergs RA (1990) The influence of exercise intensity on heat acclimation in trained subjects. Med Sci Sports Exerc 22:615-620

Jacobs RA, Rasmussen P, Siebenmann C, Díaz V, Gassmann M, Pesta D, Gnaiger E, Nordsborg NB, Robach P, Lundby C (2011) Determinants of time trial performance and maximal incremental exercise in highly trained endurance athletes. J Appl Physiol 111:1422-1430

James CA, Richardson AJ, Watt PW, Willmott AG, Gibson OR, Maxwell NS (2017) Short-term heat acclimation improves the determinants of endurance performance and 5-km running performance in the heat. Appl Physiol Nutr Metab 42:285-294

Jeukendrup AE, Hesselink MK, Snyder AC, Kuipers H, Keizer HA (1992) Physiological changes in male competitive cyclists after 2 weeks of intensified training. Int J Sports Med 13:534-541

Karlsen A, Racinais S, Jensen VM, Norgaard SJ, Bonne TC, Nybo L (2015) Heat acclimatization does not improve VO2max or cycling performance in a cool climate in trained cyclists. Scand J Med Sci Sports 25:269-276

Keiser S, Flück D, Hüppin F, Stravs A, Hilty MP, Lundby C (2015) Heat training increases exercise capacity in hot but not in temperate conditions: a mechanistic counter-balanced cross-over study. Am J Physiol Heart Circ Physiol 309:750-761

Lorenzo S, Halliwill JR, Sawka MN, Minson CT (2010) Heat acclimation improves exercise performance. J Appl Physiol 109:1140-1147 
Lorenzo S, Minson CT (2010) Heat acclimation improves cutaneous vascular function and sweating in trained cyclists. J Appl Physiol 109:1736-1743

Minson CT, Cotter JD (2016) CrossTalk proposal: heat acclimatization does improve performance in a cool condition. J Physiol 594:241-243

Nadel ER, Pandolf KB, Roberts MF, Stolwijk JA (1974) Mechanisms of thermal acclimation to exercise and heat. J Appl Physiol 37:515-520

Neal RA, Corbett J, Massey HC, Tipton MJ (2016a) Effect of shortterm heat acclimation with permissive dehydration on thermoregulation and temperate exercise performance. Scand J Med Sci Sports 26:875-884

Neal RA, Massey HC, Tipton MJ, Young JS, Corbett J (2016b) Effect of permissive dehydration on induction and decay of heat acclimation, and temperate exercise performance. Front Physiol 23:564 (eCollection)

Nielsen B, Hales JR, Strange S, Christensen NJ, Warberg J, Saltin B (1993) Human circulatory and thermoregulatory adaptations with heat acclimation and exercise in a hot, dry environment. J Physiol 460:467-485

Nielsen B, Strange S, Christensen NJ, Warberg J, Saltin B (1997) Acute and adaptive responses in humans to exercise in a warm, humid environment. Pflugers Arch 434:49-56

Nybo L, Lundby C (2016) CrossTalk opposing view: heat acclimatization does not improve exercise performance in a cool condition. J Physiol 594:245-247

Pandolf KB (1979) Effects of physical training and cardiorespiratory physical fitness on exercise-heat tolerance: recent observations. Med Sci Sports 11:60-65

Pandolf KB, Burse RL, Goldman RF (1977) Role of physical fitness in heat acclimatisation, decay and re-induction. Ergonomics 20:399-408

Périard JD, Travers GJS, Racinais S, Sawka MN (2016) Cardiovascular adaptations supporting human exercise-heat acclimation. Auton Neurosci 196:52-62

Pivarnik JM, Goetting MP, Senay LC (1987) Effect of endurance training and heat acclimation on aerobic capacity, blood volume and plasma testosterone. J Appl Sports Sci Res 1:33-35

Racinais S, Périard JD, Karlsen A, Nybo L (2015) Effect of heat and heat acclimatization on cycling time trial performance and pacing. Med Sci Sports Exerc 47:601-606

Rapp D, Scharhag J, Wagenpfeil S, Scholl J (2018) Reference values for peak oxygen uptake: cross-sectional analysis of cycle ergometrybased cardiopulmonary exercise tests of 10090 adult German volunteers from the Prevention First Registry. BMJ Open 8:e018697. https://doi.org/10.1136/bmjopen-2017-018697
Rendell RA, Prout J, Costello JT, Massey HC, Tipton MJ, Young JS, Corbett J (2017) Effects of 10 days of separate heat and hypoxic exposure on heat acclimation and temperate exercise performance. Am J Physiol Regul Integr Comp Physiol 313:191-201

Sawka MN, Leon LR, Montain SJ, Sonna LA (2011) Integrated physiological mechanisms of exercise performance, adaptation, and maladaptation to heat stress. Compr Physiol 1:1883-1928

Sawka MN, Young AJ, Cadarette BS, Levine L, Pandolf KB (1985) Influence of heat stress and acclimation on maximal aerobic power. Eur J Appl Physiol Occup Physiol 53:294-298

Schabort EJ, Killian SC, St Clair Gibson A, Hawley JA, Noakes TD (2000) Prediction of triathlon race time from laboratory testing in national triathletes. Med Sci Sports Exerc 32:844-849

Selye H (1938) Experimental evidence supporting the conception of 'adaptation energy'. Am J Physiol 123:758-765

Selye H (1950) Stress and the general adaptation syndrome. Br Med J 1:1384-1392

Senay LC, Mitchell D, Wyndham CH (1976) Acclimatization in a hot, humid environment: body fluid adjustments. J Appl Physiol 40:786-796

Shvartz E, Shapiro Y, Birnfeld H, Magazanik A (1978) Maximal oxygen uptake, heat tolerance and rectal temperature. Med Sci Sports 10:256-260

Shvartz E, Shapiro Y, Magazanik A, Meroz A, Birnfeld H, Mechtinger A, Shibolet S (1977) Heat acclimation, physical fitness, and responses to exercise in temperate and hot environments. J Appl Physiol Respir Environ Exerc Physiol 43:678-683

Sotiridis A, Debevec T, Ciuha U, Eiken O, Mekjavic IB (2018) Heat acclimation does not affect maximal aerobic power in thermoneutral normoxic or hypoxic conditions. Physiol Exp. https://doi. org/10.1113/EP087268

Taylor N, Cotter J (2006) Heat adaptation: guidelines for the optimisation of human performance. Int Sports Med J 7:33-57

Weller AS, Linnane DM, Jonkman AG, Daanen HA (2007) Quantification of the decay and re-induction of heat acclimation in dry-heat following 12 and 26 days without exposure to heat stress. Eur J Appl Physiol 102:57-66

Zhang H, Huizenga C, Arenas E, Wang D (2004) Thermal sensation and comfort in transient non-uniform thermal environments. Eur J Appl Physiol 92:728-733

Publisher's Note Springer Nature remains neutral with regard to jurisdictional claims in published maps and institutional affiliations. 